\title{
Der interessante Fall
}

Gynäkologische Endokrinologie 2020 • 18:53-56 https://doi.org/10.1007/s10304-019-00292-z

Online publiziert: 14. Januar 2020

(c) Der/die Autor(en) 2019

\section{Redaktion}

R. Felberbaum, Kempten

A. Germeyer, Heidelberg

\section{Anamnese}

Die 7-jährige Patientin wird mit einer seit 5 Tagen bestehenden vaginalen Blutung zugewiesen. Die Mutter berichtet von einem Einsetzen der Thelarche vor etwa 2 Monaten. Eine Behandlung mit Bisphosphonaten wurde aufgrund von rezidivierenden pathologischen Frakturen nach Low-impact-Belastung eingeleitet. Es sind bereits Frakturen an folgenden Extremitäten bekannt:

- Rechter Unterschenkel, distaler Humerus links, Metatarsalia II und III rechts (2015)

- Femur links, distaler Humerus rechts (2017)

\section{Befunde}

Im Rahmen der klinischen Untersuchung zeigt sich ein unauffälliges Hautbild. Das Tanner-Stadium ergibt eine Brustentwicklung bei T2-3 und eine beginnende Pubarche bei T2. In der gynäkologischen Untersuchung stellt sich die Vagina östrogenisiert dar und es zeigt sich eine leichte Schmierblutung. In der Transabdominalsonographie ist ein anteflektierter Uterus mit einem trilaminar auf $6 \mathrm{~mm}$ aufgebauten Endometrium zu sehen. Retrouterin kommt eine zystische und echoleere Struktur mit $43 \times 26 \mathrm{~mm}$ zur Darstellung (• Abb. 1).

Bei $138 \mathrm{~cm}$ Körpergröße wiegt die $\mathrm{Pa}$ tientin $29 \mathrm{~kg}$, was einem Body-Mass-Index von $15,3 \mathrm{~kg} / \mathrm{m}^{2}$ entspricht. Die Röntgenaufnahme der Handknochen ergibt eine Skelettreife von 7 Jahren und 10 Monaten. Laborchemisch zeigt sich Östradiol

Hanna Hosa' · Katharina Feil' • Bettina Böttcher ${ }^{1}$ - Elisabeth Steichen-Gersdorf ${ }^{2}$. Bettina Toth ${ }^{1} \cdot$ Beata Seeber ${ }^{1}$

' Universitätsklinik für Gynäkologische Endokrinologie und Reproduktionsmedizin, Medizinische Universität Innsbruck - Tirol Kliniken, Innsbruck, Österreich

${ }^{2}$ Department für Kinder- und Jugendheilkunde, Medizinische Universität Innsbruck, Innsbruck, Österreich

\section{Frühzeitige Pubertät und rezidivierende Frakturen}

deutlich erhöht bei Gonadotropinen unter der Nachweisgrenze (• Tab. 1).

\section{Diagnose}

In Zusammenschau der erhobenen Befunde wird die Diagnose eines atypischen McCune-Albright-Syndroms (MAS) mit Fehlen der Hautmanifestation gestellt.

\section{Verlauf}

Der Patientin wird in Abstimmung mit der pädiatrischen Endokrinologie der Aromatasehemmer Anastrozol p.o. $1 \mathrm{mg} /$ Tag verordnet, dessen Einnahme für mindestens ein Jahr bzw. bis zum normalen Pubertätseintritt fortgesetzt werden soll. Bei weiterhin leichten vaginalen Blutungen wird die laufende Medikation um Lynestrenol ergänzt, worunter es zu einem Sistieren kommt.

Bereits nach einem Monat zeigen sich laborchemisch unauffällige Hormonwerte $(17 \beta$-Östradiol $\leq 13 \mathrm{ng} / \mathrm{l})$. Die retrouterine Ovarialzyste nimmt unter der Behandlung an Größe ab (• Abb. 2) und ist im Verlauf vollständig regredient.

Bei neu auftretenden vermehrten Kopfschmerzen linksseitig zeigt eine kraniale Magnetresonanztomographie (MRT) fibröse Dysplasien an Schädelkalotte, Clivus und Schädelbasis (• Abb. 3). Zur Abklärung eines Wachstumshormonüberschusses wird ein oraler Glukosetoleranztest (oGTT) durchgeführt. Bei einer unter dem oGTT fehlenden Suppression des Wachstumshormons muss von einer überschießenden Wachstumshormonausschüttung ähnlich einer Akromegalie ausgegangen werden, weshalb eine Therapie mit einem Somatostatinanalogon s.c. $30 \mathrm{mg}$ 1-mal/Monat initiiert wird.

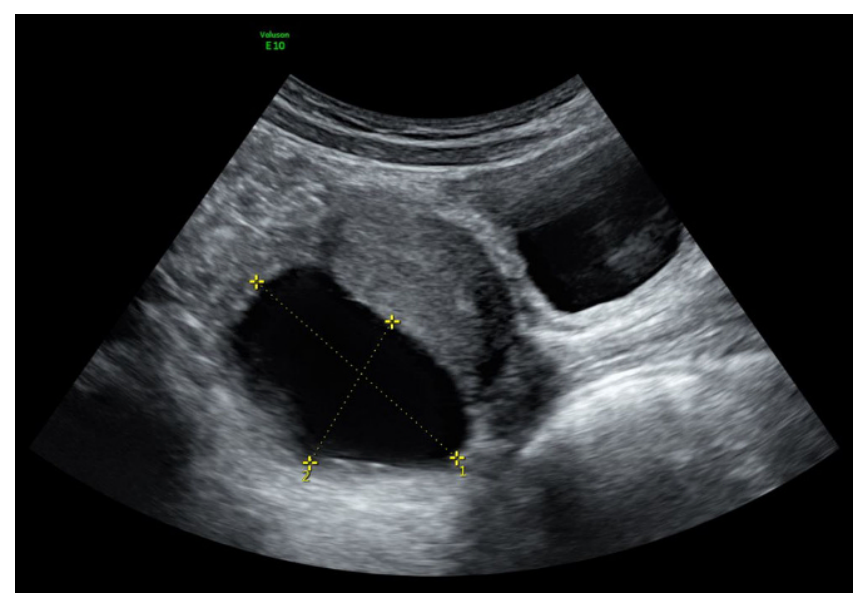

Abb. $1<$ Transabdominalsonographie: Retrouterine Ovarialzyste $43 \times 26 \mathrm{~mm}$ 
Tab. 1 Hormonwerte und Tumormarker. Blutabnahme morgens

\begin{tabular}{|c|c|c|}
\hline Parameter & Messwert & Referenzwert \\
\hline \multicolumn{3}{|l|}{ Hormone } \\
\hline Luteinisierendes Hormon (LH) & $0,1 \mathrm{U} / \mathrm{l}$ & $<0,1$ \\
\hline Follikelstimulierendes Hormon (FSH) & $<0,1 \mathrm{U} / \mathrm{l}$ & $<2,0$ \\
\hline $17 \beta-O ̈$ stradiol & $522 n g /$ & $<13-28$ \\
\hline Prolaktin & $11,8 \mu \mathrm{g} / \mathrm{l}$ & $1,2-16,0$ \\
\hline Kortisol & $44,7 \mu \mathrm{g} / \mathrm{l}$ & 06:00-10:00 48,2-195 \\
\hline Dehydroepiandrosteronsulfat (DHEAS) & $<0,30 \mathrm{mg} / \mathrm{l}$ & $0,1-0,6$ \\
\hline Gesamttestosteron & $<0,10 \mu \mathrm{g} / \mathrm{l}$ & $0,00-0,40$ \\
\hline $\begin{array}{l}\text { Thyreoideastimulierendes Hormon (TSH) } \\
\text { basal }\end{array}$ & $1,5 \mu \mathrm{U} / \mathrm{ml}$ & $0,80-5,40$ \\
\hline \multicolumn{3}{|l|}{ Tumormarker } \\
\hline hCG & $7,1 \mathrm{U} / \mathrm{I}$ & $0,0-7,0$ \\
\hline a1-Fetoprotein & $1,3 \mu \mathrm{g} / \mathrm{l}$ & $0,0-7,0$ \\
\hline $\begin{array}{l}\text { Plazentaspezifische alkalische Phosphatase } \\
\text { (AP) }\end{array}$ & $<30 \mathrm{mU} / \mathrm{l}$ & $0-100$ \\
\hline Karzinoembryonales Antigen (AG) & $0,8 \mu \mathrm{g} / \mathrm{l}$ & $0,0-3,8$ \\
\hline CA-125 & $13 \mathrm{kU} / \mathrm{l}$ & $0-35$ \\
\hline$C A_{\text {"Cancer antigen" }}$ & & \\
\hline
\end{tabular}

\section{Diskussion}

\section{Typische Trias}

Charakterisierend für das MAS ist folgende typische Trias:

- Polyostotische fibröse Dysplasie: bindegewebiger Umbau in den einem gesteigerten Remodeling unterliegenden Geflechtknochen. Es kann zu schmerzhaften Deformierungen, Wachstumsstörungen und pathologischen Frakturen kommen. Die Erstmanifestation der pathologischen Frakturen liegt primär zwischen dem fünften und zehnten Lebensjahr [1].

- Periphere Pubertas praecox

- Irreguläre Café-au-Lait-Flecken: meist ohne Überschreiten der Mittellinie, den Blaschko-Linien folgend

\section{Pathogenese}

Pathogenetisch liegt eine spontane postzygotische Mutation des Gens GNAS1 (Chromosom 20) vor. Dieser Genlocus codiert das $\mathrm{G}_{\mathrm{s}}-\alpha$-Protein, das an der Regulation von zyklischem Adenosinmonophosphat (cAMP) beteiligt ist. Bei Betroffenen kommt es zu einer cAMP-Hochregulierung mit einer nachfolgend kontinuierlichen Stimulation der endokrinen Regelkreise. Der
Mutationszeitpunkt in der Embryogenese ist entscheidend für die Ausprägung des genetischen Mosaiks, weshalb es neben der genannten Trias auch zu einem atypischen MAS kommen kann.

\section{Manifestation}

Die periphere Pubertas praecox ist die häufigste Manifestation im Rahmen des Syndroms [1, 2]. Sind weitere endokrine Organe beteiligt, zeigt sich eine Hyperthyreose, ein renaler Phosphatverlust, ein Wachstumshormonexzess oder ein aktive Organe wie die Leber und das Herz können mitklinisch imponierender Cholestase, Hepatitis oder kardialen Arrhythmien betroffen sein.

\section{》) Auch nicht endokrin aktive Organe wie die Leber und das Herz können betroffen sein}

Die Patientinnen entwickeln oftmals rezidivierende funktionelle Ovarialzysten, die aufgrund der Östradiolsekretion zu einer Östrogenisierung des Endometriums und nachfolgend zu vaginalen Blutungen führen können. Die Prävalenz des MAS beträgt $1: 100.000$ bis $1: 1.000 .000$ [3]. Cushing-Syndrom. Auch nicht endokrin

\section{Therapie}

Therapie der Wahl ist die Gabe von Inhibitoren der gonadalen Sexualsteroidproduktion bzw. ihrer Zielrezeptoren. Um die Fertilität zu erhalten, sollte von einem operativen Vorgehen bezüglich der Ovarialzysten Abstand genommen werden.

Bei peripherer Pubertas praecox wird eine Therapie mit Aromataseinhibitoren wie Letrozol oder Anastrozol („off label“) empfohlen. Diese führen zu einer Reduktion der vaginalen Blutungsepisoden und der damit einhergehenden psychischen Belastung sowie zu einer Abnahme der Knochenalterung bzw. einer Verhinderung des frühzeitigen Epiphysenfugenschlusses. Im Falle von weiterhin bestehenden Blutungen kann eine Therapieergänzung mit einem Gestagenpräparat in ausreichender Transformationsdosis in Erwägung gezogen werden. Da Lynestrenol - wie im dargestellten Fall beschrieben - aktuell nicht erhältlich ist, kann alternativ zum Beispiel Chlormadinonacetat $2 \mathrm{mg}$, Medroxyprogesteronacetat 5-10 mg oder Dydrogesteron $10 \mathrm{mg} 2$-mal täglich gegeben werden. Die Gabe von Antiöstrogenen wie Tamoxifen wird aufgrund einer uterinen Volumenzunahme [2] und entsprechender Risiken bei Langzeitanwendung kontrovers diskutiert. Während der Therapie sind regelmäßige Verlaufskontrollen indiziert, da sich bei einer länger andauernden Exposition gegenüber Sexualsteroiden auch eine zentrale Pubertas praecox entwickeln kann.

\section{Pubertas praecox}

Eine Pubertätsentwicklung in einem Alter von 7 Jahren wird als Pubertas praecox bezeichnet, wobei auch die Familienanamnese, das Körpergewicht und die Ethnizität berücksichtigt werden sollen.

Wichtig ist folgende Unterscheidung:

- Zentrale Pubertas praecox, wobei es gonadotropinabhängig zu einer frühzeitigen Reifung der Hypothalamus-Hypophysen-GonadenAchse kommt. Die entstehenden Geschlechtsmerkmale sind isosexuell.

- Periphere Pubertas praecox, die gonadotropinunabhängig bei einer 


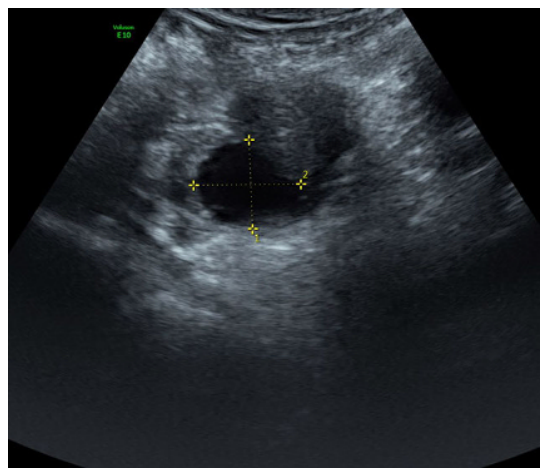

Abb. 2 ム Transabdominalsonographie. Retrouterine Ovarialzyste $23 \times 19 \mathrm{~mm}$

erhöhten Ausschüttung von Östrogenen bzw. Androgenen aus Gonaden oder Nebennieren, einer exogenen Zufuhr von Sexualsteroiden oder einem hormonproduzierenden Tumor eine frühzeitige Entwicklung von isosexuellen oder kontrasexuellen Geschlechtsmerkmalen zeigt.

- Isolierte Adrenarche/Thelarche/ benigne Normvariante bedingt durch eine frühzeitige Aktivierung der $\mathrm{Ne}$ bennieren- oder der HypothalamusHypophysen-Gonaden-Achse.

Wegweisend sind die Anamnese in Bezug auf das Auftreten von Thelarche, Pubarche, Menarche oder Wachstumsschübe sowie eine Erhebung der Tanner-Stadien.

Bei Verdacht auf einen Tumor werden Inhibin und humanes Choriongonadotropin bestimmt

Eine Röntgenuntersuchung der linken Hand dient der Bestimmung des biologischen Knochenalters. Zur Diagnostik gehören eine erweiterte Hormonbasisdiagnostik mit zusätzlicher Bestimmung von 17-Hydroxyprogesteron (17-OHP), Dehydroepiandrosteronsulfat und Androstendion. In Abhängigkeit der Befunde kann ein Gonadotropin-ReleasingHormon-Test veranlasst werden, der der Unterscheidung zwischen einer peripheren und zentralen Pubertas praecox dient. Bei Verdacht auf das Vorliegen eines Tumors sollten Inhibin und humanes Choriongonadotropin bestimmt werden.

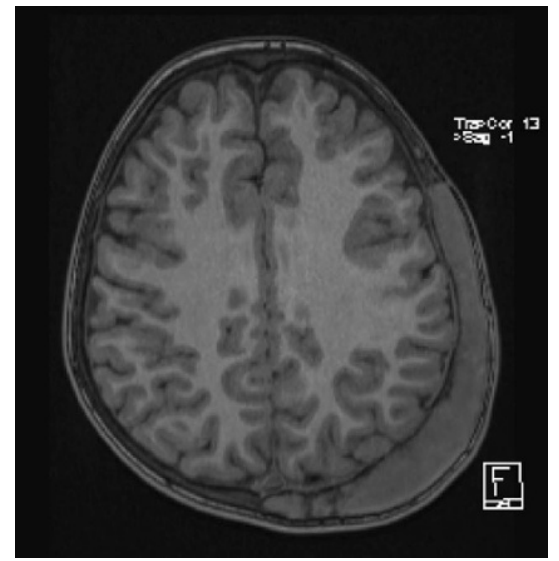

Abb. 3 A Magnetresonanztomographie des Schädels. Fibröse Dysplasie links parietal. (- Abb. 3 wurde dankenswerterweise von der Universitätsklinik für Radiologie, Innsbruck, zur Verwendung im Rahmen der Fallpräsentation zur Verfügung gestellt)

Die bildgebenden Verfahren werden je nach Verdachtsdiagnose angeschlossen. Bildmorphologisch gibt eine Transabdominalsonographie Aufschluss über das Volumen der Ovarien und des Uterus, das Vorliegen von Zysten, die Höhe des Endometriums und etwaige Raumforderungen. Bei Verdacht auf einen zerebralen oder adrenalen Tumor erfolgt die weitere Diagnostik mittels Kontrastmittel-MRT.

Als Differenzialdiagnosen einer peripheren Pubertas praecox sind funktionelle Ovarialzysten und Tumoren wie Granulosazelltumoren (isosexuelle Frühreifung) oder Sertoli-LeydigZell-Tumoren, Leydig-Zell-Tumoren und Gonadoblastome (kontrasexuelle Frühreifung) zu nennen. Bei exogen zugeführten Sexualsteroiden, adrenalen Störungen wie dem adrenogenitalen Syndrom oder Tumoren der Nebenniere sowie bei Vorliegen einer schweren primären Hypothyreose kann es zu einer peripheren Pubertas praecox kommen.

\section{Fazit für die Praxis}

\section{- Die Unterscheidung zwischen iso-} lierter Thelarche/Adrenarche sowie einer zentralen und peripheren $\mathrm{Pu}$ bertas praecox ist wegweisend für die Behandlungsmodalität.
- Die Behandlung erfolgt interdisziplinär gemeinsam mit der pädiatrischen Endokrinologie.

- Bei Vorliegen von Ovarialzysten sollten konservative Therapieoptionen operativen vorgezogen werden.

- Es erfolgt ein Therapieversuch mit Aromataseinhibitoren (,,off label“) für mindestens ein Jahr oder bis zum altersadäquaten Pubertätseintritt. Klinische, laborchemische und sonographische Kontrollen werden engmaschig durchgeführt.

\section{Korrespondenzadresse}

\section{Dr. Katharina Feil}

Universitätsklinik für Gynäkologische Endokrinologie und Reproduktionsmedizin, Medizinische Universität Innsbruck - Tirol Kliniken

Anichstraße 35, 6020 Innsbruck, Österreich katharina.feil@tirol-kliniken.at

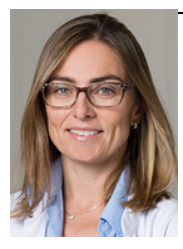

Prof. Dr. Beata Seeber Universitätsklinik für Gynäkologische Endokrinologie und Reproduktionsmedizin, Medizinische Universität Innsbruck - Tirol Kliniken Anichstraße 35, 6020 Innsbruck, Österreich beata.seeber@i-med.ac.at

Funding. Open access funding provided by University of Innsbruck and Medical University of Innsbruck.

\section{Einhaltung ethischer Richtlinien}

Interessenkonflikt. H. Hosa, K. Feil, B. Böttcher, E. Steichen-Gersdorf, B. Toth und B. Seeber geben an, dass kein Interessenkonflikt besteht.

Für diesen Beitrag wurden von den Autoren keine Studien an Menschen oder Tieren durchgeführt. Für die aufgeführten Studien gelten die jeweils dort angegebenen ethischen Richtlinien. Für Bildmaterial oder anderweitige Angaben innerhalb des Manuskripts, über die Patienten zu identifizieren sind, liegt von ihnen und/oder ihren gesetzlichen Vertretern eine schriftliche Einwilligung vor.

Open Access. Dieser Artikel wird unter der Creative Commons Namensnennung 4.0 International Lizenz veröffentlicht, welche die Nutzung, Vervielfältigung, Bearbeitung, Verbreitung und Wiedergabe in jeglichem Medium und Format erlaubt, sofern Sie den/die ursprünglichen Autor(en) und die Quelle ordnungsgemäß nennen, einen Link zur Creative Commons Lizenz beifügen und angeben, ob Änderungen vorgenommen wurden.

Die in diesem Artikel enthaltenen Bilder und sonstiges Drittmaterial unterliegen ebenfalls der genannten 


\section{Der interessante Fall}

Creative Commons Lizenz, sofern sich aus der Abbildungslegende nichts anderes ergibt. Sofern das betreffende Material nicht unter der genannten Creative Commons Lizenz steht und die betreffende Handlung nicht nach gesetzlichen Vorschriften erlaubt ist, ist für die oben aufgeführten Weiterverwendungen des Materials die Einwilligung des jeweiligen Rechteinhabers einzuholen.

Weitere Details zur Lizenz entnehmen Sie bitte der Lizenzinformation auf http://creativecommons.org/ licenses/by/4.0/deed.de.

\section{Literatur}

1. Hou JW, Syndrome MC-A (2018) Diagnosis and clinical course in eleven patients. Pediatr Neonatol 59(4):418-420

2. Eugster EA, Rubin SD et al (2003) Tamoxifen treatment for precocious puberty in McCuneAlbright syndrome: a multicenter trial. J Pediatr 143(1):60-66

3. Dumitrescu CE et al (2008) McCune-Albright syndrome. Orphanet J Rare Dis 3:12

Hier steht eine Anzeige.

\section{黛 Springer}

